\title{
High-density lipoprotein cholesterol efflux capacity is inversely associated with cardiovascular risk: a systematic review and meta-analysis
}

\author{
Chengfeng Qiu ${ }^{1,2,3}$, Xiang Zhao ${ }^{4}$, Quan Zhou ${ }^{5}$ and Zhen Zhang ${ }^{2,6^{*}}$
}

\begin{abstract}
Background: A low plasma level of high-density lipoprotein ( $\mathrm{HDL}$ ) cholesterol $(\mathrm{HDL}-\mathrm{C})$ is associated with cardiovascular risk. A key cardioprotective property of HDL is cholesterol efflux capacity (CEC), the ability of HDL to accept cholesterol from macrophages. In this study, we aimed to identify the predictive value of CEC for cardiovascular risk.

Methods: The relative risks (RRs) and 95\% confidence intervals (Cls) were pooled to analyze the association between CEC and the incidence of cardiovascular events and all-cause mortality. The odds ratios (ORs) and 95\% Cls were pooled to estimate the association of CEC and the prevalence of cardiovascular events.

Results: A total of 15 studies were included. Results showed that the highest CEC was significantly associated with a reduced risk of cardiovascular events incidents compared to the lowest CEC (RR, $0.56 ; 95 \% \mathrm{Cl}, 0.37$ to $\left.0.85 ; P^{2}, 89 \%\right)$; the pooled RR of cardiovascular risk for per unit SD increase was $0.87\left(95 \% \mathrm{Cl}, 0.73\right.$ to $1.04 ; \ell^{2}$, 67\%). Dose-response curve indicated that cardiovascular risk decreased by $39 \%$ (RR, $0.61 ; 95 \% \mathrm{Cl}, 0.51$ to 0.74$)$ for per unit CEC increase. Similarly, an inverse association was observed between CEC and the prevalence of cardiovascular events (highest vs. lowest, OR, 0.30; $95 \% \mathrm{Cl}, 0.17$ to $0.5 ; P^{2}=63 \%$; per unit SD increase, $\mathrm{OR}, 0.94 ; 95 \% \mathrm{Cl}, 0.90$ to $\left.0.98 ; P^{2}=71 \%\right)$. However, based on the current data, CEC was not significantly associated with all-cause mortality.

Conclusions: Findings from this meta-analysis suggest that HDL-mediated CEC is inversely associated with cardiovascular risk, which appears to be independent of HDL concentration. The growing understanding of CEC and its role in cardiovascular risk decrease may improve the accuracy of cardiovascular risk prediction and also open important avenues to develop novel therapeutic targeting HDL metabolism.
\end{abstract}

Keywords: High-density lipoprotein, Cholesterol efflux capacity, Cardiovascular risk, Meta-analysis

\section{Background}

High-density lipoprotein (HDL) cholesterol (HDL-C) is ubiquitously regarded as the "good cholesterol", and its complex relationship with cardiovascular risk has been a topic of great interest for decades. Epidemiologic studies revealed a robust and inverse association between low HDL-C and cardiovascular risk [1]. This association gave rise to the hypothesis that "higher HDL is better".

\footnotetext{
* Correspondence: js200_1986@163.com

${ }^{2}$ Center for Vascular Disease and Translational Medicine, The Third Xiangya Hospital of Central South University, Changsha, Hunan 410013, China

${ }^{6}$ Centre for Experimental Medicine, Third Xiangya Hospital of Central South University, Changsha, Hunan 410013, China

Full list of author information is available at the end of the article
}

However, the hypothesis has been challenged recently on the basis of clinical and genetic studies. Pharmacological interventions such as niacin and cholesteryl ester transfer protein (CETP) inhibitors, increased HDL-C but failed to reduce cardiovascular risk [2-5]. Genetic studies in humans also revealed that certain genetic variants associated with increased HDL-C do not protect the carriers against cardiovascular disease (CVD) [6]. HDL particles vary in lipid and protein composition and HDL subspecies serve different biological functions [7]; thus, HDL concentration does not accurately reflect the biological function. This suggests the limited value of using steady-state HDL concentrations to assess the cardioprotective effects of HDL. 
HDLs exert several activities to provide protection against the development of atherosclerosis; these functions are related to reverse cholesterol transport (RCT) as well as anti-inflammatory, endothelial and vascular functions [8]. Among them, RCT is considered to be the key cardioprotective property of HDL. RCT is the complex process by which HDLs accept cholesterol derived from artery-wall macrophages and mediate delivery to the liver for disposition [8,9]. Macrophages laden with excessive cholesteryl esters form foam cells, which are implicated in the causal pathway of atherosclerosis [10, 11]. By functioning as an acceptor of cholesterol derived from macrophages, HDLs can inhibit and/or reduce foam cell formation. Cholesterol efflux from macrophages is the initial step of RCT and the cholesterol efflux capacity (CEC) is commonly used to assess the biological function of HDLs in ex vivo [7].

In recent years, ex vivo CEC assays have been successfully used in clinical studies to measure the capacity of apolipoprotein B (apoB)-depleted serum to accept labeled cholesterol from macrophage cells [12]. Although recent studies have been conducted to investigate the association between CEC and cardiovascular risk, the results of these studies are inconsistent.

Accordingly, in this study, we performed a standard meta-analysis and dose-response analysis to estimate the association between CEC and cardiovascular risk to identify the predictive value of CEC for cardiovascular risk. This information is important in guiding the development of the HDL-targeted therapies that reduce cardiovascular risk.

\section{Methods}

This study was performed according to the guidelines proposed by the Meta-analysis of Observational Studies in Epidemiology (MOOSE) group. The Research plan was defined prior to conducting the review.

\section{Date sources and extraction}

Studies of the relationship between HDL-mediated CEC and cardiovascular risk were considered to be eligible for inclusion. Two investigators independently searched for the relevant studies in the MEDLINE, EMBASE and Cochrane Library databases. Literature searches were last updated on February 1st, 2017. The following groups of key words were used in literature searches: "cholesterol efflux capacity" OR "HDL-mediated cholesterol efflux", and "cardiovascular events" OR "mortality" OR "all-cause death" OR "cardiovascular risk" OR "acute coronary syndromes" OR "myocardial infarction" OR "cardiovascular disease" OR "risk" OR "death" OR "mortality" OR "outcome" OR "stroke" OR "transient ischemic attacks" OR "intracranial hemorrhage" OR "events". An additional manual search of the reference lists of original articles and review articles was also performed.
Two investigators independently conducted the study selection on the basis of predefined criteria by reviewing the abstract and full-text. Studies that reported the association between HDL-mediated CEC and human cardiovascular risk were included in the initial study selection process. Outcomes of included studies were identified as the incidence and prevalence of cardiovascular events, and all-cause mortality. Cardiovascular events were defined as a composite of atherosclerotic cardiovascular events and death from cardiovascular causes. If multiple studies researched the same population, the study with the most detailed data or the larger sample size was selected. Studies were excluded if they failed to meet all the listed inclusion criteria. One investigator extracted data, while a second checked the data for accuracy. All disagreements were resolved in consultation with a third investigator.

The following information was extracted from each of the eligible studies: first author's name, publication year, country, sample size, clinical characteristics of participants, age, male/female, follow-up time, CEC assay methods, CEC level, events, effect size and corresponding 95\% CI, adjusted factors.

\section{CEC assay methods}

Ex vivo CEC assays, which are readily used in clinical studies, consist of three components are involved: labeled cholesterol, a donor cell which releases the labeled cholesterol, and a cholesterol acceptor, which are prepared from clinical serum samples using standard methods. To reduce the variation between studies, three elements of CEC assays were identified as follows: (i) donor cell lines were macrophages (J774 cells or THP-1 cells); (ii) cholesterol was radiolabeled with ${ }^{3} \mathrm{H}$ or labeled with fluorescence; (iii) and serum samples from clinical subjects which were processed to remove apoB were used as cholesterol acceptors.

\section{Quality assessment}

Two investigators independently assessed the quality of each study by applying the criteria defined by the Newcastle-Ottawa Scale (NOS) score, which is widely used in the assessment of the quality of observational studies. Three factors comprising selection, comparability and outcome are used in the assessment criteria, and the scores range from 0 to 9.

\section{Data synthesis and analysis}

In the current meta-analysis, we used relative risk (RR) with corresponding $95 \%$ confidence interval (CI) as a measure of the effect size to evaluate the association between HDLmediated CEC and the incidence of cardiovascular events and all-cause mortality. The effect size was calculated based on comparison of the highest versus the lowest CEC, plus 1 SD CEC increase. Multivariable-adjusted RRs or hazard 
ratios (HRs) of the original studies were pooled. The odds ratios (ORs) and 95\% CIs were pooled to estimate the association between CEC and the prevalence of cardiovascular events. For the dose-response meta-analysis, we used the "generalized least squares for trend estimation" method proposed by Greenland and Longnecker $[13,14]$ to take into account the correlation with the $\log R R$ estimates across the levels of CEC. This method requires knowledge of the cases and cohort size/control subjects of each category and the RR with its variance estimate for at least three quantitative exposure categories. The value assigned to each level of cholesterol efflux capacity was the median/ mean provided by original research. We estimated the potential dose-response relationship in two-stages. In the first stage, we estimated a restricted cubic spline model with three knots at percentiles 10, 50 and $90 \%$ of the distribution of levels of cholesterol efflux capacity. In the second stage, the two regression coefficients (3 knots minus 1 ) and the variance/covariance matrix within each study were combined in a multivariate random-effects metaanalysis. A $P$-value for nonlinearity was calculated by testing the null hypothesis that the coefficient of the second spline is equal to 0 .

Between-study heterogeneity was assessed by the chisquared-based Q test and $I^{2}$ statistics [15]. A $P$-value $<0.10$ for the $\mathrm{Q}$ test and $I^{2}>50$ were considered to indicate significant heterogeneity. Publication bias was assessed by visual inspection of funnel plots for asymmetry and statistical evaluation with Begg's rank correlation test [16] and Egger's linear regression test [17]. Two-tailed $\alpha$ level of significance was set at 0.05 .

Statistical analyses were performed with STATA/SE.12.0 (StataCorp, College station, Texas, USA), Review Manager Version 5.3 (The Nordic Cochrane Center, Copenhagen, Denmark) and R version 3.2.0 (R Foundation for Statistical Computing, Vienna, Austria).

\section{Results}

Baseline characteristics and quality of included studies

Figure 1 shows the study selection process. A total of 140 articles were considered to be potentially relevant studies after comprehensive searches of the PubMed, EMBASE and Cochrane library databases. A total of 15 articles with 16,364 participants identified by full-text reviews were included in the final meta-analysis. The ages of participants ranged from 42 to 72 years, and 55\% (9027) were males. We included 12 cohort studies from nine published articles $(n=13,754)$ to evaluate the association between CEC and the incidence of cardiovascular events; the median followup was 5.6 years. In addition, four studies reporting the multivariable-adjusted HR or RR for the incidence of cardiovascular events with different categories of CEC, were

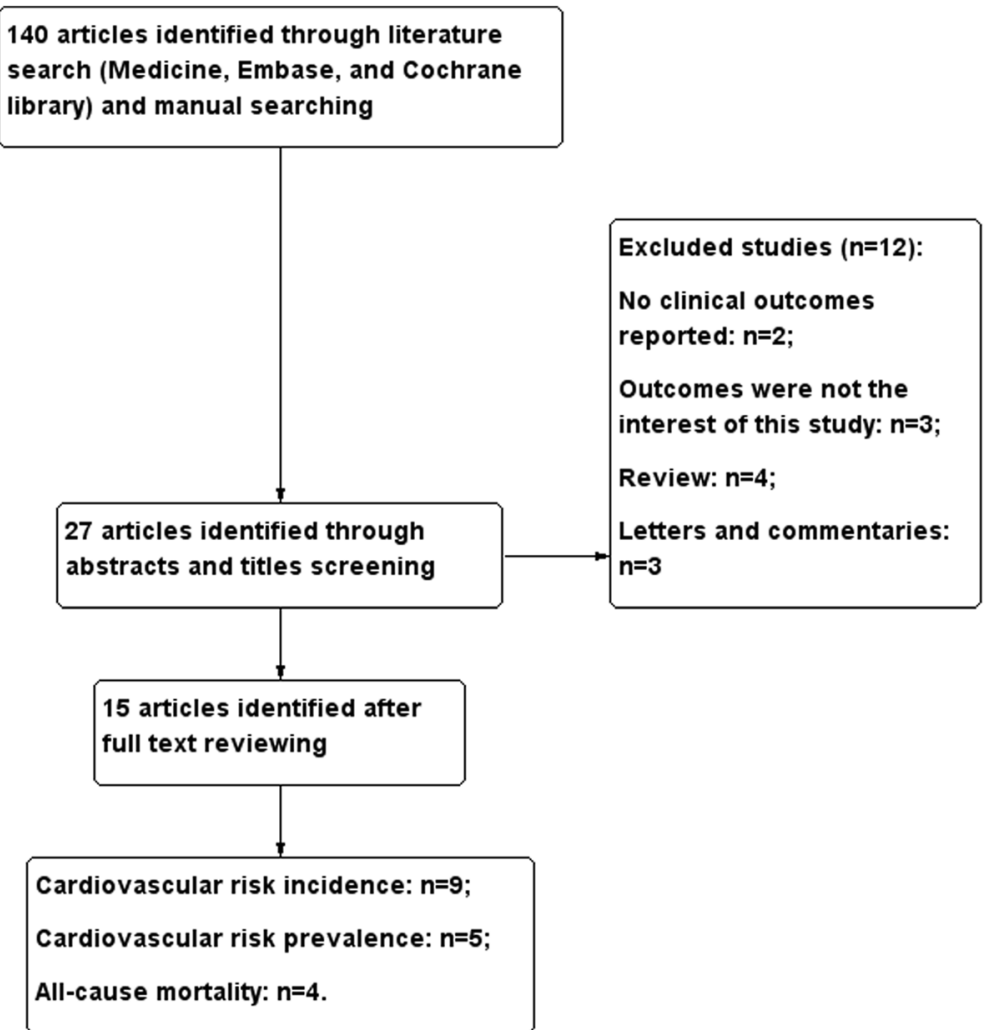

Fig. 1 Flow diagram of study selection process 
included in the dose-response analysis. Eight studies from five articles $(n=4732)$ were included to evaluate the prevalence of cardiovascular events and five studies from four articles $(n=3940)$ were included to assess the all-cause mortality risk. The quality of the included studies was assessed by NOS; scores ranged from 5 to 9 (Table 1 and Additional file 1). Full details of the baseline characteristics of the included articles are presented in Table 1. Events and effect sizes (HR or RR or OR) of original studies are listed in Table 2.

\section{The association between HDL-mediated CEC and the incidence of cardiovascular events}

When compared to the lowest CEC, the highest levels of CEC were significantly associated with reduced cardiovascular risk (13,259 participants across 8 studies; pooled RR, 0.56; $95 \%$ CI, 0.37 to $0.85 ; I^{2}, 89 \%$; Fig. 2). No publication bias was found via visual inspection of funnel plots for asymmetry (Additional file 2) and statistical evaluation with Begg's and Egger's tests ( $P$-value for Begg's and Egger's tests were 0.39 and 0.11 , respectively). The pooled RR of the incidence of cardiovascular events for 1 SD increase was 0.87 (6869 participants across 4 studies, $95 \% \mathrm{CI}, 0.73$ to 1.04 ; $I^{2}, 67 \%$; Fig. 2 ), and no obvious publication bias was observed (Additional file 2; $P$ value for Begg's and Egger's tests were 0.308 and 0.388, respectively). Sensitivity analysis was performed to confirm the robustness of these findings by sequential application of the leave-one-out method to investigate the influence of each individual study on the overall risk estimate. The results did not show any significant change in the pooled effect size (Additional files 3 and 4).

Subgroup analysis stratified by clinical characteristics of subjects showed a strong association of CEC with the incidence of cardiovascular events in healthy individuals (highest vs. lowest, RR, $0.38 ; 95 \% \mathrm{CI}, 0.28$ to $0.52 ; I^{2}=0$; Fig. 3) and in patients with baseline CVD (highest vs. lowest, RR, 0.23; 95\% CI, 0.13 to $0.40 ; I^{2}=0$; Fig. 3). However, such an inverse relationship was not demonstrated in the patients with renal impairment (highest vs. lowest, RR, 0.92; 95\% CI, 0.83 to $1.02 ; I^{2}=0$; per $1 \mathrm{SD}$ increase, RR, 0.94; 95\% CI, 0.86 to $1.04 ; I^{2}=0$; Fig. 3). Subgroup analysis stratified by ethnicity demonstrated consistent results among Asian populations (highest vs. lowest, RR, 0.23 ; 95\% CI, 0.13 to $0.40 ; I^{2}=0$; per $1 \mathrm{SD}$ increase, RR, 0.08; 95\% CI, 0.01 to $0.66 ; I^{2}=0$; Fig. 3), and among European populations (highest vs. lowest, RR, $0.71 ; 95 \%$ CI, 0.50 to $1.00 ; I^{2}=81$; per 1 SD increase, RR, 0.88 ; 95\% CI, 0.78 to $1.00 ; I^{2}=52$; Fig. 3).

Four studies provided data for the dose-response metaanalysis. Using a restricted cubic spline model, we observed a linear dose-response association between CEC and cardiovascular risk $\left[\mathrm{x}^{2}\right.$ test for non-linearity $=2.2,(\mathrm{df}=2)$, $P=0.33]$. The dose-response curve (Fig. 4) indicated that the cardiovascular risk decreased by $39 \%$ (RR, $0.61 ; 95 \% \mathrm{CI}$, 0.51 to 0.74$)$ per unit increment in cholesterol efflux capacity.

\section{The association between HDL-mediated CEC and the prevalence of cardiovascular events}

Compared with the lowest CEC, the prevalence of cardiovascular events was significantly reduced in the participants with the highest CEC (OR, 0.30; 95\% CI, 0.17 to $0.51 ; I^{2}=63 \%$; Fig. 3). Pooled results showed a slight reduction in the prevalence of cardiovascular events with per 1 SD increase (OR, 0.94; 95\% CI, 0.90 to $0.98 ; I^{2}=71 \%$; Fig. 5). Potential publication bias was assessed by visual inspection of funnel plots for asymmetry (Additional file 5) and through an Egger's linear regression test with a $P$-value of 0.005 . Results from sensitivity analysis did not show a significant change (Additional files 6 and 7).

\section{The association between HDL-mediated CEC and all-cause mortality}

In the final meta-analysis, a total of 6824 participants were included to evaluate the association between CEC and allcause mortality. There was no statistically significant difference in the pooled effect size in the comparisons of the highest with lowest CEC, as well as in the comparisons of the data per 1 SD increase (Fig. 6). Visual inspection of funnel plots for asymmetry indicated the potential existence of publication bias (Additional file 8).

\section{Discussion}

To the best of our knowledge, the is the first comprehensive evaluation of the association between HDL-mediated CEC and cardiovascular risk performed by meta-analysis. Three important results were found in this study. First, there was a strong inverse association between HDLmeditated CEC and the incidence of cardiovascular events, especially in healthy individuals and patients with CVD at baseline. Dose-response analysis demonstrated a linear, dose-dependent association, while the cardiovascular risk decreased as the increased CEC. Second, CEC was also inversely associated with the prevalence of cardiovascular events. Third, although there was a trend toward an inverse association between HDL-CEC and all-cause mortality, this was not found to be statistically significant based on the current data.

HDLs comprise various subspecies and vary widely in particle size, as well as the lipid and protein composition [18]. Indeed, it is now widely known that specific HDL subspecies exert highly specific functions $[9,19]$. Static concentration measurements are poorly reflecting the biologic activities of HDL [20]. This may account for the failure of pharmacologic interventions (niacin and CETP inhibitors) designed to raise plasma HDL-cholesterol 


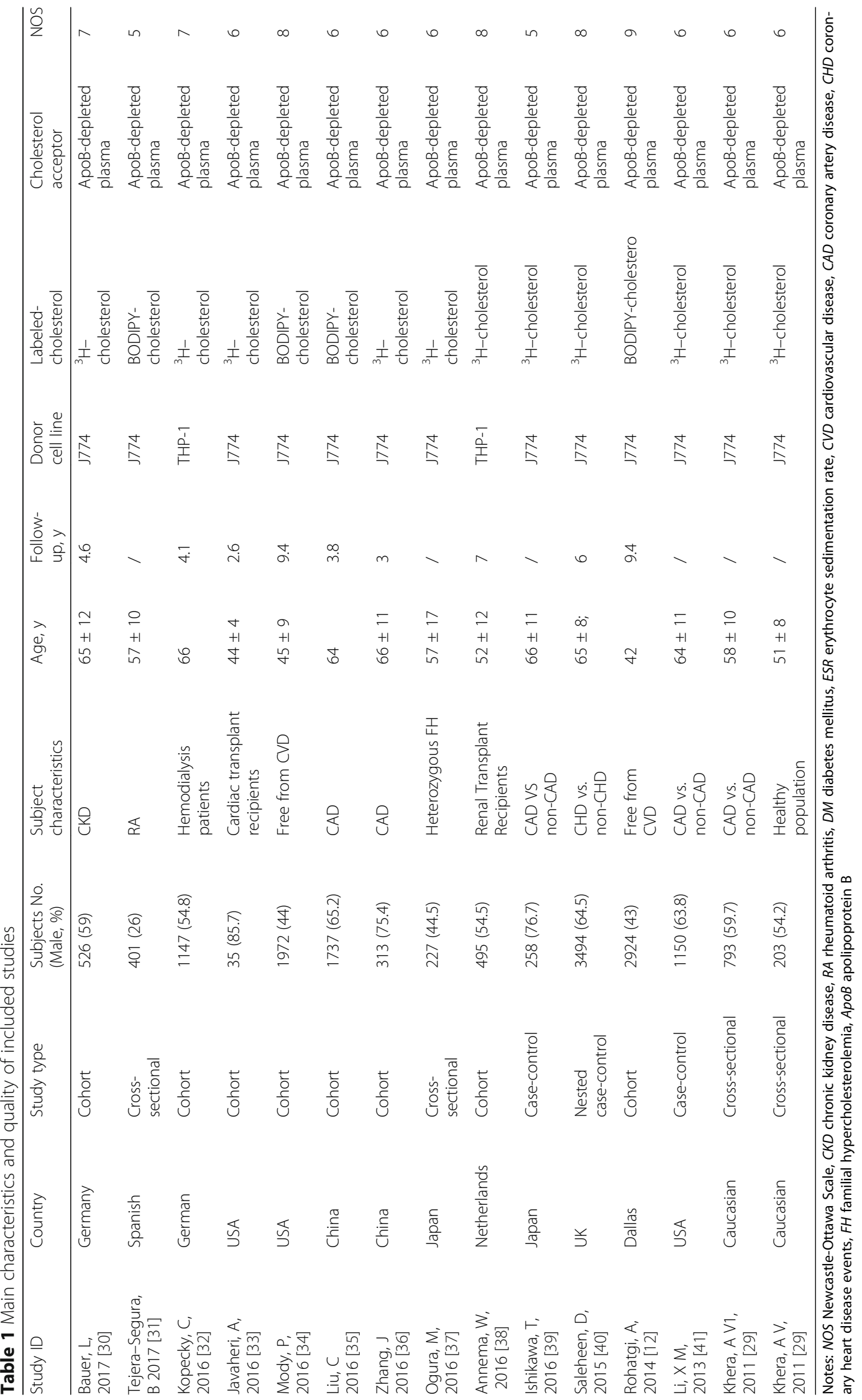


Table 2 Effect sizes of included studies in this meta-analysis

\begin{tabular}{|c|c|c|c|c|}
\hline Study ID & $\begin{array}{l}\text { CEC } \\
\text { (median/mean) }\end{array}$ & Events & $\mathrm{HR} / \mathrm{RR} / \mathrm{OR}(95 \% \mathrm{Cl})$ & Adjustment factors \\
\hline $\begin{array}{l}\text { Bauer, L } \\
2017[30]\end{array}$ & $12.2 \pm 2.4$ & CVE & $\begin{array}{l}\text { Q1: } 1 ; \\
\text { Q2: } 0.58(0.33-1.00) ; \\
\text { Q3: } 0.67(0.39-1.15) ; \\
\text { Q4: } 0.91(0.51-1.62) ;\end{array}$ & $\begin{array}{l}\text { Age, sex, BMI, BP, smoking status, GFR, } \\
\text { and log albuminuria }\end{array}$ \\
\hline $\begin{array}{l}\text { Tejera-Segura, } \\
\text { B } 2017 \text { [31] }\end{array}$ & $\begin{array}{l}\text { Control: } 16.9 \pm 10.4 \\
\text { Case: } 18.9 \pm 9.0\end{array}$ & $\begin{array}{l}\text { Subclinical } \\
\text { atherosclerosis }\end{array}$ & $0.94(0.89-0.98)$ & $\begin{array}{l}\text { Age, sex, SBP, DM, ESR, DAS28 and } \\
\text { tocilizumab use }\end{array}$ \\
\hline $\begin{array}{l}\text { Kopecky, } \\
\text { C, } 2016[32]\end{array}$ & $\begin{array}{l}\text { T1: } 0.73 \\
\text { T2: } 0.89 \\
\text { T3: } 1.08\end{array}$ & CVD & $0.92(0.83-1.02)$ & $\begin{array}{l}\text { Traditional risk factors, LDL-C, HDL-C, } \\
\text { apoA-I, and CRP }\end{array}$ \\
\hline Javaheri, A, 2016 [33] & $\begin{array}{l}\text { Alive: } 0.98 \pm 0.03 \\
\text { Dead: } 0.89 \pm 0.03\end{array}$ & Mortality & $0.19(0.06-0.56)$ & $\begin{array}{l}\mathrm{HDL}-\mathrm{C}, \mathrm{LDL}-\mathrm{C} \text {, ischemic origin, and } \\
\text { rejection }\end{array}$ \\
\hline $\begin{array}{l}\text { Mody, } \\
\text { P, } 2016[34]\end{array}$ & / & CVD & $0.35(0.23-0.55)$ & $\begin{array}{l}\mathrm{TC}, \mathrm{HDL}-\mathrm{C} \text {, history of blood pressure } \\
\text { medication use, BMI, and CRP }\end{array}$ \\
\hline $\begin{array}{l}\text { Liu, C } \\
2016[35]\end{array}$ & $\begin{array}{l}\text { Q1: } 0.70(0.17-0.79) ; \\
\text { Q2: } 0.86(0.79-0.93) ; \\
\text { Q3: } 1.00(0.93-1.07) ; \\
\text { Q4: } 1.15(1.07-2.01)\end{array}$ & $\begin{array}{l}\text { All-cause death and } \\
\text { cardiovascular death }\end{array}$ & $\begin{array}{l}\text { All-cause death: } \\
0.10(0.01-0.74) ; \\
\text { Cardiovascular death: } \\
0.08(0.01-0.68)\end{array}$ & $\begin{array}{l}\text { Age, sex, BMl, smoking and alcohol } \\
\text { drinking, hypertension, DM, dyslipidemia, } \\
\text { lipid-lowering drug use, TC, TG, LDL-C, } \\
\mathrm{HDL}-\mathrm{C} \text {, and apoA-I }\end{array}$ \\
\hline $\begin{array}{l}\text { Zhang, J } \\
2016 \text { [36] }\end{array}$ & / & CVD & $0.30(0.14-0.67)$ & $\begin{array}{l}\text { Age, sex, hypertension, diabetes, current } \\
\text { smoking, } L D L-C, H D L-C, A p o A, A p o B, \\
\text { and regular medication }\end{array}$ \\
\hline $\begin{array}{l}\text { Ogura, M, } \\
2016[37]\end{array}$ & / & Incidence of CVD & $0.95(0.90-0.99)$ & $\begin{array}{l}\text { Age, sex, hypertension, diabetes mellitus, } \\
\text { smoking history, obesity, LDL-C, TG, HDL-C }\end{array}$ \\
\hline Annema, W, 2016 [38] & $\begin{array}{l}\text { T1 (\%): 5.8(5.3-6.4); } \\
\text { T2 (\%): 7.3(6.8-7.9); } \\
\text { T3 (\%): } 9.0(8.2-9.8)\end{array}$ & $\begin{array}{l}\text { All-cause death and } \\
\text { cardiovascular death }\end{array}$ & $\begin{array}{l}\text { CV mortality: } \\
0.96 \text { (0.72-1.27); } \\
\text { All-cause mortality: } \\
0.84 \text { (0.68-1.04) }\end{array}$ & $\begin{array}{l}\text { Age, sex, apo A-I, HDL-C cholesterol and } \\
\text { creatinine clearance }\end{array}$ \\
\hline $\begin{array}{l}\text { Ishikawa, } \\
\text { T, } 2016[39]\end{array}$ & $\begin{array}{l}\text { CAD: } 0.86 \pm 0.26 \\
\text { Non-CAD: } 1.02 \pm 0.38\end{array}$ & Incidence of CVD & $0.23(0.056-0.91)$ & Baseline adjustment \\
\hline Saleheen, D, 2015 [40] & / & Incidence of CHD events & $\begin{array}{l}\text { Top vs. bottom: } \\
0.64(0.51-0.80) ; \\
\text { Per } 1 \text { SD: } \\
0.80(0.70-0.90)\end{array}$ & $\begin{array}{l}\text { Age, sex, diabetes, hypertension, cigarette } \\
\text { use, alcohol use, waist:hip ratio and BMI, } \\
\text { LDL-C, TG and HDL-C }\end{array}$ \\
\hline $\begin{array}{l}\text { Rohatgi, } \\
\text { A, } 2014 \text { [12] }\end{array}$ & $0.21-3.93$ & Incidence of CVD & $0.33(0.109-0.55)$ & $\begin{array}{l}\text { Age, sex, race, diabetes, hypertension, } \\
\text { smoking, BMI, TG, TC, and statin use }\end{array}$ \\
\hline $\begin{array}{l}\mathrm{Li}, \times \mathrm{M} \\
2013[41]\end{array}$ & / & Incidence of CAD and MACE & $1.85(1.11-3.06)$ & $\begin{array}{l}\text { Age, sex, smoking, diabetes mellitus, } \\
\text { hypertension, LDL-C, and HDL-C }\end{array}$ \\
\hline $\begin{array}{l}\text { Khera, A } \\
\text { V, } 2011[29]\end{array}$ & $\begin{array}{l}\text { Case: } 0.82 \\
\text { Control: } 0.9\end{array}$ & CAD & $\begin{array}{l}\text { Per } 1 \text { SD increase: } \\
0.75(0.63-0.90) ; \\
\text { Q4 vs. Q1: } \\
0.48(0.30-0.78)\end{array}$ & Cardiovascular risk factors and $\mathrm{HDL}-\mathrm{C}$ \\
\hline $\begin{array}{l}\text { Khera, A } \\
\text { V, } 2011[29]\end{array}$ & $0.77(0.36-1.68)$ & CVD prevalence & $\begin{array}{l}\text { Per } 1 \text { SD increase: } \\
0.97(0.94-0.99)\end{array}$ & $\begin{array}{l}\text { Age, sex, cardiovascular risk factors a } \\
\text { nd HDL-C }\end{array}$ \\
\hline
\end{tabular}

Notes: $C E C$ cholesterol efflux capacity, $R R$ risk ratio, $H R$ hazard ratio, $C l$ confidence interval, $O R$ odds ratio, NOS Newcastle-Ottawa Scale, $C K D$ chronic kidney disease, $C V E$ cardiovascular event, $B M I$ body mass index, $B P$ blood pressure, SBP systolic blood pressure, GFR glomerular filtration rate, $R A$ rheumatoid arthritis, DM diabetes mellitus, ESR erythrocyte sedimentation rate, CVD cardiovascular disease, $L D L-C$ low density lipoprotein-cholesterol, $H D L-C$ high-density lipoprotein-cholesterol, $A p o$ apolipoprotein, CRP C-reactive Protein, $T C$ total cholesterol, CAD coronary artery disease, $Q$ quartile, $T G$ triglyceride, $C H D$ coronary heart disease events, $T$ tertiles, MACE major adverse cardiovascular event

levels to improve cardiovascular outcomes. HDL-mediated cholesterol efflux from macrophages, which is the key functional property of HDL in protecting against atherosclerosis, is a critical process that is evaluated by RCT $[7,21$, 22]. In recent years, a growing number of studies have focused on HDL function, especially CEC, instead of simply assessing HDL concentrations, with the aim of gaining an improved understanding of the clinical benefits of HDL in protecting against CVD. However, these clinical studies have yielded inconsistent results, and the link between HDL-mediated CEC and cardiovascular risk remains to be fully clarified.

Importantly, analysis of the pooled results in this study showed that the capacity of HDL to promote cholesterol efflux was inversely associated with the future incidence of cardiovascular events. Furthermore, the dose-response 


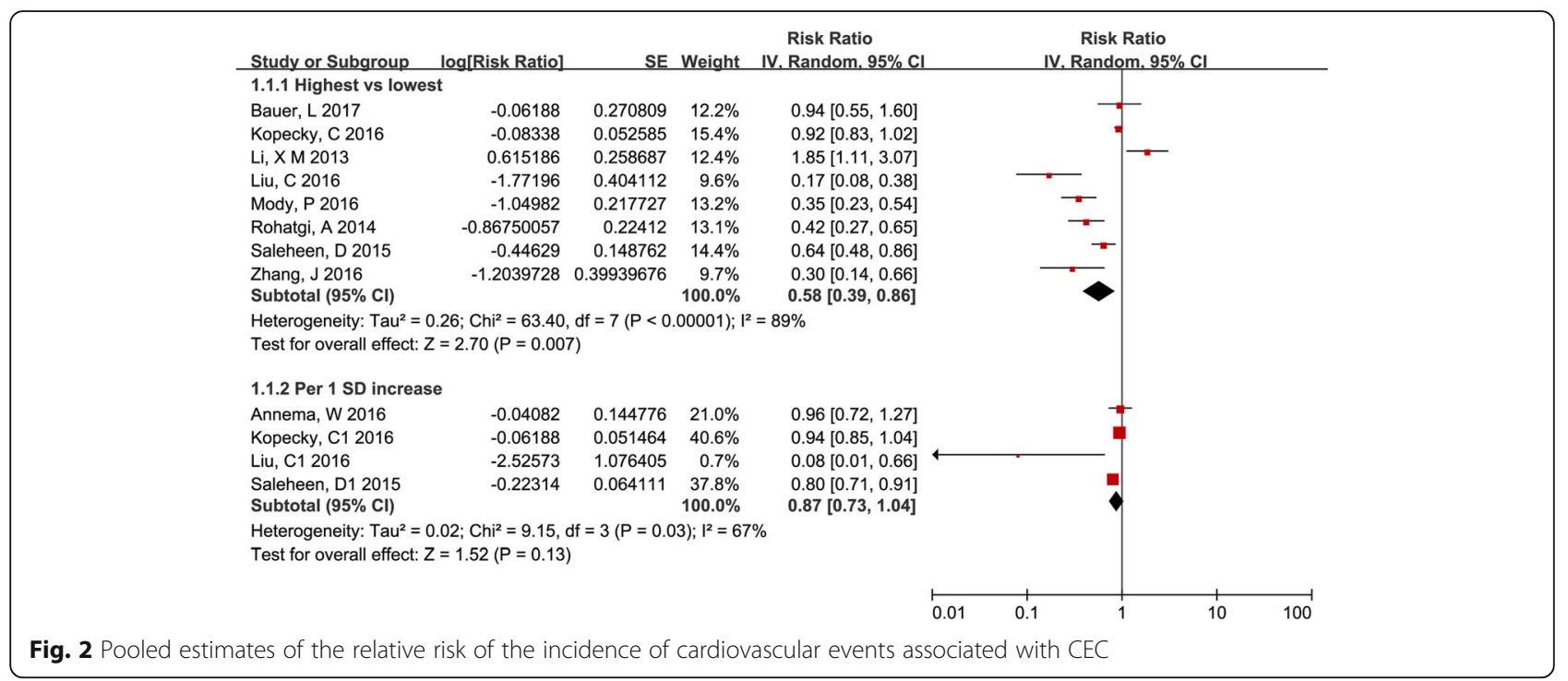

analysis demonstrated that increased CEC was related to decreased cardiovascular risk, in a relationship that is apparently independent of HDL levels. In the subgroup analysis stratified by ethnicity, significant inverse associations between CEC and cardiovascular risk were observed both in European and Asian populations. The pooled effect size (RR) was much smaller in Asian populations than that in European populations. It is well recognized that there are differences in cardiovascular risk between European and Asian populations involving many factors, including genetic factors, lifestyle, and environment. Studies have demonstrated that lifestyle changes also influence CEC [23]. Nevertheless, we were unable to identify ethnicity as a determinant factor that may influence the predictive value of CEC for cardiovascular risk, because of limited data. This is an interesting issue that warrants further investigation. Further subgroup analysis stratified according to the baseline clinical characteristics of participants revealed that the results are influenced by disease. First, we found that HDL-cholesterol efflux capacity was a strong predictor of cardiovascular risk both in healthy individuals and in patients with CVD. As an integrated measure of HDL quantity and quality, CEC accurately reflects the role of HDL in atheroprotection. Studies have demonstrated that HDL-cholesterol efflux exerts multiple cardioprotective properties, the most important being that the cholesterol efflux from macrophages inhibits foam cell formation and protects macrophages from LDL-induced apoptosis [24]. In addition, CEC has been shown to be an important signaling pathway required for nitric oxide activation and protein transport, and enhances endothelial function by mediating anti-inflammatory and anti-oxidant

\begin{tabular}{|c|c|c|c|c|c|c|}
\hline Subgroup & Sample & HR( $(95 \% \mathrm{CI})$ & & $\mathbf{P}^{*}$ & $I^{2}(\%)$ & $\mathbf{P}_{\text {het }}$ \\
\hline \multicolumn{7}{|l|}{ Participant } \\
\hline \multicolumn{7}{|l|}{ Healthy people } \\
\hline Highest vs lowest & 4896 & $0.38(0.28,0.52)$ & $\mapsto-1$ & $<0.00$ & 0 & 0.56 \\
\hline \multicolumn{7}{|l|}{ Patients with CVD } \\
\hline Highest vs lowest & 2050 & $0.23(0.130 .40)$ & H一 & $<0.00$ & 0 & 0.32 \\
\hline Per 1 SD increase & 5227 & $0.33(0.04,2.94)$ & $\mathrm{H}$ & 0.32 & 78 & 0.03 \\
\hline \multicolumn{7}{|l|}{ Patients with CKD } \\
\hline Highest vs lowest & 1673 & $0.92(0.83,1.02)$ & $\mapsto$ & 0.11 & 0 & 0.94 \\
\hline Per 1 SD increase & 1642 & $0.94(0.86,1.04)$ & H. & 0.22 & 0 & 0.89 \\
\hline \multicolumn{7}{|l|}{ Ethnicity } \\
\hline \multicolumn{7}{|l|}{ European } \\
\hline Highest vs lowest & 8087 & $0.71(0.50,1.00)$ & . & 0.05 & 81 & $<0.00$ \\
\hline Per 1 SD increase & 5132 & $0.88(0.78,1.00)$ & r. & 0.05 & 52 & 0.12 \\
\hline \multicolumn{7}{|l|}{ Asian } \\
\hline Highest vs lowest & 2050 & $0.23(0.13,0.40)$ & $\mathrm{H}-\mathrm{C}$ & $<0.00$ & 0 & 0.32 \\
\hline Per 1 SD increase & 1737 & $0.08(0.01,0.66)$ & Hב- & 0.02 & & \\
\hline
\end{tabular}

Fig. 3 Subgroup analyses of the relative risk of the incidence of cardiovascular events stratified by clinical characteristics of the participant and ethnicity. Notes: $P_{\text {het }}$ was utilized to assess the between-study heterogeneity using the chi-squared-based $Q$ test 


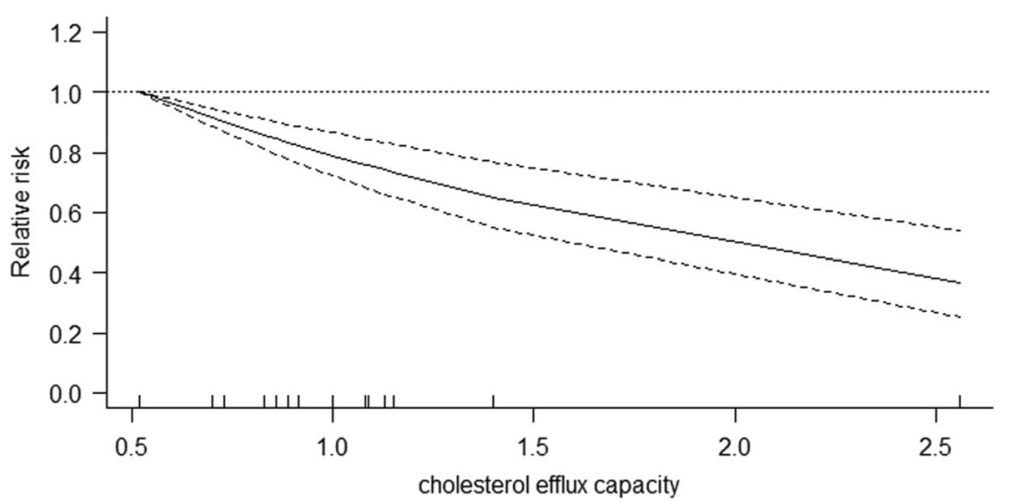

Fig. 4 Dose-response association between CEC and cardiovascular risk incidence

activities [25, 26]. Accordingly, increased CEC enhances the cardioprotective activities of HDL, and vice versa. Although limited data were pooled in this meta-analysis and statistical heterogeneity was present, these results provide important information showing that HDLmediated macrophage-specific CEC is potentially a stronger predictor of cardiovascular risk among patients with various CVDs and healthy individual. However, CEC was not correlated with cardiovascular risk in the patients with kidney disease. The potential underlying reason for this could be the dysfunction of HDL in kidney disease. HDL particles are characterized by an altered molecular composition under conditions of kidney disease, which is not restricted to the anti-inflammatory and anti-oxidative effects, but also result in decreased CEC [27]. Moreover, these patients usually lose lipoproteins via the urine and as a consequence, it is likely that not only the quality, but also the quantity of HDL is decreased. In fact, cardiovascular risk is notably increased in individuals with chronic kidney disease, mainly due to the high prevalence of traditional risk factors, such as hypertension, diabetes, dyslipidemia and high levels of inflammation [28]. The findings of this study showed that disease characteristics affect the predictive value of CEC for cardiovascular risk. HDLmediated CEC may be a good predictive biomarker of cardiovascular risk in healthy individuals and in patients with CVD, but not in patients with impaired kidney function.

A previous cross-sectional study reported a strong inverse association between CEC and the prevalence of coronary artery disease [29]. A similar association was found between CEC and the prevalence of cardiovascular risk in this study. Because of the obvious heterogeneity and potential publication bias, the association was weaker and remains to be confirmed by more clinical studies. However, our results did not show a statistical association between CEC and all-cause mortality. This indicates that HDL-mediated CEC is predominantly correlated with cardiovascular risk, but not with the other risks. The small sample $(n=6824)$ included in this meta-analysis and the existence of heterogeneity may reduce the credibility of this result.

The findings of our study may have important clinical implications. Although cholesterol efflux from macrophages represents only a small component of the overall turnover of cholesterol in the body as a

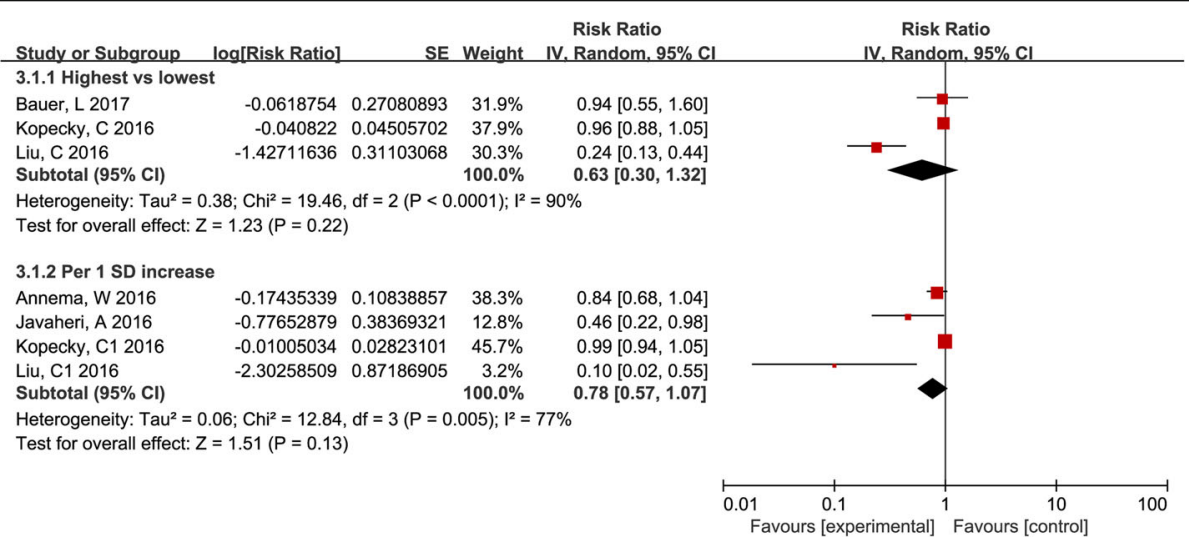

Fig. 5 Pooled estimates of the odds ratios of the prevalence of cardiovascular events associated with CEC 


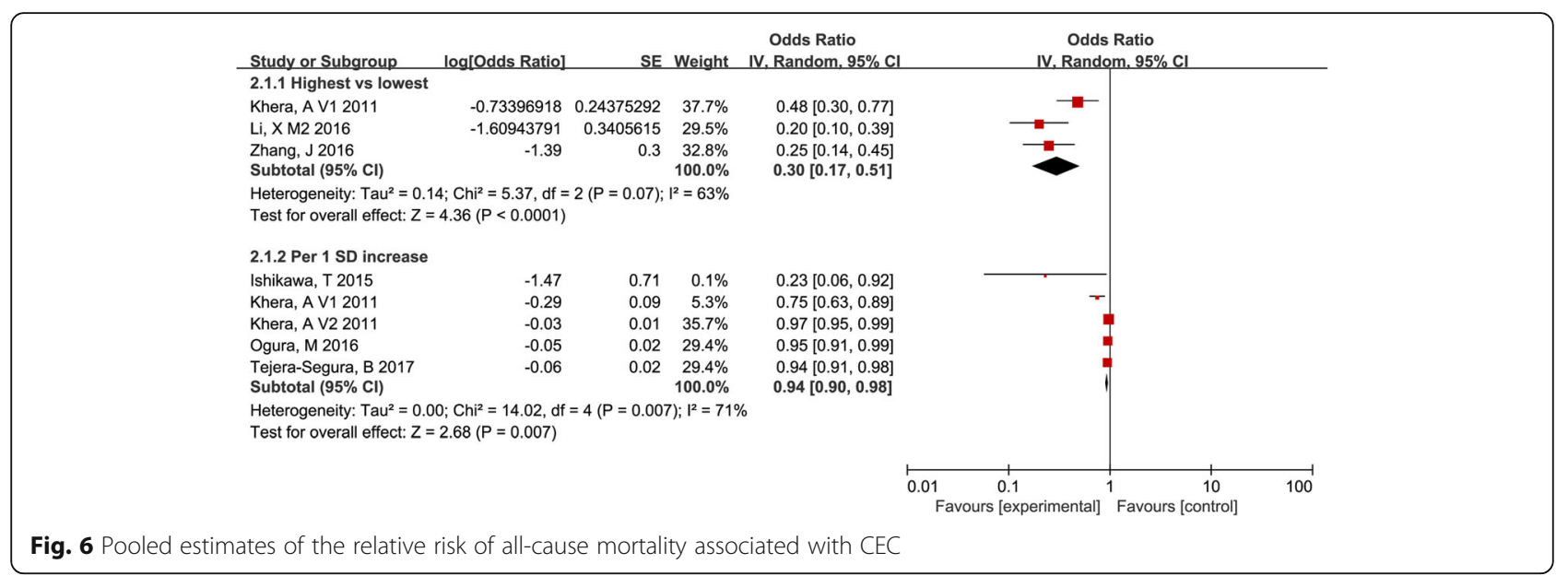

whole, it is probably the property that is most relevant to atheroprotection and accurately reflects HDL function. The results of this study suggest a potential role of HDL-mediated CEC in cardiovascular risk prediction and support the use of CEC assays in guiding the development of new HDL-targeted therapies. However, before the measurement of CEC is widely recommended in clinical practice, it is necessary to standardize CEC assays and develop high-throughput platforms that are suitable for routine clinical use.

Several limitations of this study should be recognized. First, combined cardiovascular events were identified as the outcome to estimate the cardiovascular risk in the included studies, thus, making it difficult to identify the risk of specific cardiovascular events, such as myocardial infarctions and ischemic strokes. Larger clinical trials dealing the association of CEC with the incidence of specific cardiovascular events are urgently needed to validate this concept. Second, the pooled results were based on observational studies, and although multiple-adjusted effect size was selected, the adjusted factors were not consistent. Hence, we cannot exclude the impact of the other confounders, which may account for some between-study variation. Finally, the most important limitation is that there is no established gold-standard for ex vivo CEC assays. For example, CEC measurement using different forms of labeled cholesterol (radiolabel or fluorescence label) and differences in donor cell lines (J774 or THP-1) can yield between-study variation. At present, the cholesterol efflux assay is not readily applicable in routine clinical practice. Our findings support the implementation of efforts to establish a standardized CEC assay that is suitable for clinical use.

\section{Conclusions}

In conclusion, the findings of this meta-analysis suggest that HDL-mediated cholesterol efflux capacity is inversely associated with the cardiovascular risk, which appears to be independent of HDL concentrations. A significant linear association revealed that the cardiovascular risk decreased as the CEC increased. The growing understanding of CEC and its role in cardiovascular risk decrease may improve the accuracy of risk prediction and also open important avenues to develop novel therapeutic targeting HDL metabolism.

\section{Additional files}

Additional file 1: Quality assessment of included studies. (XLS $36 \mathrm{~kb}$ )

Additional file 2: Funnel plot of CEC and the incidence of cardiovascular events. (TIFF $4376 \mathrm{~kb}$ )

Additional file 3: Sensitivity analysis of the association between CEC and the incidence of cardiovascular events (highest vs. lowest CEC). (TIFF $9883 \mathrm{~kb}$ )

Additional file 4: Sensitivity analysis of the association between CEC and the incidence of cardiovascular events with 1 SD increase in CEC. (TIFF $9883 \mathrm{~kb}$ )

Additional file 5: Funnel plot of CEC and the prevalence of cardiovascular events. (TIFF $7496 \mathrm{~kb}$ )

Additional file 6: Sensitivity analysis of the association between CEC and the prevalence of cardiovascular events (highest vs. lowest CEC). (TIFF $6331 \mathrm{~kb}$ )

Additional file 7: Sensitivity analysis of the association between CEC and the prevalence of cardiovascular events with 1 SD increase in CEC. (TIFF $6332 \mathrm{~kb}$ )

Additional file 8: Funnel plot of CEC and all-cause mortality. (TIFF $4376 \mathrm{~kb})$

\section{Abbreviations}

Apo: Apolipoprotein; BMl: Body mass index; BP: Blood pressure; CAD: Coronary artery disease; CEC: Cholesterol efflux capacity;

CETP: Cholesteryl ester transfer protein; CHD: Coronary heart disease events; Cl: Confidence interval; CKD: Chronic kidney disease; CRP: C-Reactive Protein; CVD: Cardiovascular disease; CVE: Cardiovascular event; DM: Diabetes mellitus; ESR: Erythrocyte sedimentation rate; GFR: Glomerular filtration rate; HDL-C: High-density lipoprotein-cholesterol; HR: Hazard ratio; LDL-C: Low density lipoprotein-cholesterol; MACE: Major adverse cardiovascular event; MOOSE: Meta-analysis of Observational Studies in Epidemiology; NOS: Newcastle-Ottawa Scale; OR: Odds ratios; Q: Quartile; RA: Rheumatoid arthritis; RCT: Reverse cholesterol transport; RR: Relative risk; SBP: Systolic blood pressure; T: Tertiles; TC: Total cholesterol; TG: Triglyceride 


\section{Acknowledgments}

Not applicable.

\section{Funding}

This study was supported by the National Science Foundation of China (NSFC) Project 81,600,247, the New Xiangya Talent Projects of the Third Xiangya hospital of Central South University JY201626, and the Natural Science Foundation of Hunan Province, China 2017JJ3250.

\section{Availability of data and materials}

Data are available from the authors on request.

\section{Authors' contributions}

ZZ and QCF conceived and designed the research, acquired the data, performed statistical analysis, drafted, revised and approved the manuscript submitted. ZX and QCF carried out data extraction and quality assessment; ZQ performed the dose-response analysis. All authors read and approved the final manuscript.

\section{Ethics approval and consent to participate} Not applicable.

\section{Consent for publication}

Not applicable.

\section{Competing interests}

The authors declare that they have no competing interests.

\section{Publisher's Note}

Springer Nature remains neutral with regard to jurisdictional claims in published maps and institutional affiliations.

\section{Author details}

${ }^{1}$ Xiangya school of Pharmaceutical Sciences, Central South University, Changsha, Hunan 410013, China. ${ }^{2}$ Center for Vascular Disease and Translational Medicine, The Third Xiangya Hospital of Central South University, Changsha, Hunan 410013, China. ${ }^{3}$ Department of Pharmacy, The First People's Hospital of Huaihua City, Huaihua 418000, China. ${ }^{4}$ Department of Emergency, The First People's Hospital of Huaihua City, Huaihua, Hunan 418000, China. ${ }^{5}$ Department of Science and Education, The First People's Hospital of Changde City, Changde, Hunan 415003, China. ${ }^{6}$ Centre for Experimental Medicine, Third Xiangya Hospital of Central South University, Changsha, Hunan 410013, China.

Received: 7 September 2017 Accepted: 2 November 2017 Published online: 10 November 2017

\section{References}

1. Stone NJ, Robinson JG, Lichtenstein AH, Bairey MC, Blum CB, Eckel RH, et al. 2013 ACC/AHA guideline on the treatment of blood cholesterol to reduce atherosclerotic cardiovascular risk in adults: a report of the American College of Cardiology/American Heart Association Task Force on Practice Guidelines. Circulation. 2014;129(25 Suppl 2):S1-45.

2. Landray MJ, Haynes R, Hopewell JC, Parish S, Aung T, Tomson J, et al. Effects of extended-release niacin with laropiprant in high-risk patients. N Engl J Med. 2014;371(3):203-12

3. Schwartz GG, Olsson AG, Abt M, Ballantyne CM, Barter PJ, Brumm J, et al. Effects of dalcetrapib in patients with a recent acute coronary syndrome. N Engl J Med. 2012:367(22):2089-99.

4. Boden WE, Probstfield JL, Anderson T, Chaitman BR, Desvignes-Nickens P, Koprowicz $\mathrm{K}$, et al. Niacin in patients with low HDL cholesterol levels receiving intensive statin therapy. N Engl J Med. 2011;365(24):2255-67.

5. Barter PJ, Caulfield M, Eriksson M, Grundy SM, Kastelein JJ, Komajda M, et al. Effects of torcetrapib in patients at high risk for coronary events. N Engl J Med. 2007;357(21):2109-22

6. Voight BF, Peloso GM, Orho-Melander M, Frikke-Schmidt R, Barbalic M, Jensen MK, et al. Plasma HDL cholesterol and risk of myocardial infarction: a mendelian randomisation study. Lancet. 2012;380(9841):572-80.

7. Anastasius M, Kockx M, Jessup W, Sullivan D, Rye KA, Kritharides L. Cholesterol efflux capacity: an introduction for clinicians. Am Heart J. 2016; 180:54-63.
8. Rosenson RS, Brewer HJ, Chapman MJ, Fazio S, Hussain MM, Kontush A, et al. HDL measures, particle heterogeneity, proposed nomenclature, and relation to atherosclerotic cardiovascular events. Clin Chem. 2011;57(3):392-410.

9. Rosenson RS, Brewer HB, Davidson WS, Fayad ZA, Fuster V, Goldstein J, et al. Cholesterol efflux and atheroprotection: advancing the concept of reverse cholesterol transport. Circulation. 2012;125(15):1905-19.

10. Moore K, Tabas I. Macrophages in the pathogenesis of atherosclerosis. Cell. 2011;145(3):341-55.

11. Moore KJ, Sheedy FJ, Fisher EA. Macrophages in atherosclerosis: a dynamic balance. Nat Rev Immunol. 2013;13(10):709-21.

12. Rohatgi A, Khera A, Berry JD, Givens EG, Ayers CR, Wedin KE, et al. HDL cholesterol efflux capacity and incident cardiovascular events. N Engl J Med. 2014;371(25):2383-93.

13. Greenland S, Longnecker MP. Methods for trend estimation from summarized dose-response data, with applications to meta-analysis. Am J Epidemiol. 1992;135(11):1301-9.

14. Greenland S. Quantitative methods in the review of epidemiologic literature. Epidemiol Rev. 1987;9:1-30.

15. Higgins JP, Thompson SG. Quantifying heterogeneity in a meta-analysis. Stat Med. 2002;21(11):1539-58.

16. Begg $C B$, Mazumdar M. Operating characteristics of a rank correlation test for publication bias. Biometrics. 1994;50(4):1088-101.

17. Egger M, Davey SG, Schneider M, Minder C. Bias in meta-analysis detected by a simple, graphical test. BMJ. 1997;315(7109):629-34.

18. Vaisar T, Pennathur S, Green PS, Gharib SA, Hoofnagle AN, Cheung MC, et al. Shotgun proteomics implicates protease inhibition and complement activation in the antiinflammatory properties of HDL. J Clin Invest. 2007;117(3):746-56.

19. Davidson WS, Silva RA, Chantepie S, Lagor WR, Chapman MJ, Kontush A. Proteomic analysis of defined HDL subpopulations reveals particle-specific protein clusters: relevance to antioxidative function. Arterioscler Thromb Vasc Biol. 2009;29(6):870-6.

20. de la Llera-Moya M, Drazul-Schrader D, Asztalos BF, Cuchel M, Rader DJ, Rothblat $\mathrm{GH}$. The ability to promote efflux via ABCA1 determines the capacity of serum specimens with similar high-density lipoprotein cholesterol to remove cholesterol from macrophages. Arterioscler Thromb Vasc Biol. 2010;30(4):796-801.

21. Hutchins PM, Heinecke JW. Cholesterol efflux capacity, macrophage reverse cholesterol transport and cardioprotective HDL. Curr Opin Lipidol. 2015; 26(5):388-93.

22. Rohatgi A. High-density lipoprotein function measurement in human studies: focus on cholesterol efflux capacity. Prog Cardiovasc Dis. 2015;58(1):32-40.

23. Blazek A, Rutsky J, Osei K, Maiseyeu A, Rajagopalan S. Exercise-mediated changes in high-density lipoprotein: impact on form and function. Am Heart J. 2013;166(3):392-400.

24. Rader DJ, Alexander ET, Weibel GL, Billheimer J, Rothblat GH. The role of reverse cholesterol transport in animals and humans and relationship to atherosclerosis. J Lipid Res. 2009;50(Suppl):S189-94.

25. Mineo C, Shaul PW. Regulation of signal transduction by HDL. J Lipid Res. 2013;54(9):2315-24.

26. Rader DJ, Hovingh GK. HDL and cardiovascular disease. Lancet. 2014; 384(9943):618-25.

27. Weichhart T, Kopecky C, Kubicek M, Haidinger M, Doller D, Katholnig K, et al. Serum amyloid A in uremic HDL promotes inflammation. J Am Soc Nephrol. 2012;23(5):934-47.

28. Gansevoort RT, Correa-Rotter R, Hemmelgarn BR, Jafar TH, Heerspink HJ, Mann JF, et al. Chronic kidney disease and cardiovascular risk: epidemiology, mechanisms, and prevention. Lancet. 2013;382(9889):339-52.

29. Khera AV, Cuchel M, de la Llera-Moya M, Rodrigues A, Burke MF, Jafri K, et al. Cholesterol efflux capacity, high-density lipoprotein function, and atherosclerosis. N Engl J Med. 2011;364(2):127-35.

30. Bauer L, Kern S, Rogacev KS, Emrich IE, Zawada A, Fliser D, et al. HDL cholesterol efflux capacity and cardiovascular events in patients with chronic kidney disease. J Am Coll Cardiol. 2017;69(2):246-7.

31. Tejera-Segura B, Macia-Diaz M, Machado JD, de Vera-Gonzalez A, GarciaDopico JA, Olmos JM, et al. HDL cholesterol efflux capacity in rheumatoid arthritis patients: contributing factors and relationship with subclinical atherosclerosis. Arthritis Res Ther. 2017;19(1):113.

32. Kopecky C, Ebtehaj S, Genser B, Drechsler C, Krane V, Antlanger M, et al. $\mathrm{HDL}$ cholesterol efflux does not predict cardiovascular risk in hemodialysis patients. J Am Soc Nephrol. 2016;3(28):769-75.

33. Javaheri A, Molina M, Zamani P, Rodrigues A, Novak E, Chambers S, et al. Cholesterol efflux capacity of high-density lipoprotein correlates with 
survival and allograft vasculopathy in cardiac transplant recipients. J Heart Lung Transplant. 2016;11(35):1295-302.

34. Mody P, Joshi PH, Khera A, Ayers CR, Rohatgi A. Beyond coronary calcification, family history, and C-reactive protein: cholesterol efflux capacity and cardiovascular risk prediction. J Am Coll Cardiol. 2016;67(21):2480-7.

35. Liu C, Zhang Y, Ding D, Li X, Yang Y, Li Q, et al. Cholesterol efflux capacity is an independent predictor of all-cause and cardiovascular mortality in patients with coronary artery disease: a prospective cohort study. Atherosclerosis. 2016;249:116-24.

36. Zhang J, Xu J, Wang J, Wu C, Xu Y, Wang Y, et al. Prognostic usefulness of serum cholesterol efflux capacity in patients with coronary artery disease. Am J Cardiol. 2016;117(4):508-14.

37. Ogura M, Hori M, Harada-Shiba M. Association between cholesterol efflux capacity and atherosclerotic cardiovascular disease in patients with familial hypercholesterolemia. Arterioscler Thromb Vasc Biol. 2016;36(1):181-8.

38. Annema W, Dikkers A, de Boer JF, Dullaart RP, Sanders JS, Bakker SJ, et al. $\mathrm{HDL}$ cholesterol efflux predicts graft failure in renal transplant recipients. J Am Soc Nephrol. 2016;27(2):595-603.

39. Ishikawa T, Ayaori M, Uto-Kondo H, Nakajima T, Mutoh M, Ikewaki K. Highdensity lipoprotein cholesterol efflux capacity as a relevant predictor of atherosclerotic coronary disease. Atherosclerosis. 2015;242(1):318-22.

40. Saleheen D, Scott R, Javad S, Zhao W, Rodrigues A, Picataggi A, et al. Association of $\mathrm{HDL}$ cholesterol efflux capacity with incident coronary heart disease events: a prospective case-control study. Lancet Diabetes Endocrinol. 2015;3(7):507-13.

41. Li XM, Tang WHW, Mosior MK, Huang Y, Wu Y, Matter W, et al. Paradoxical association of enhanced cholesterol efflux with increased incident cardiovascular risks. Arterioscler Thromb Vasc Biol. 2013;33(7):1696-705.

\section{Submit your next manuscript to BioMed Central and we will help you at every step:}

- We accept pre-submission inquiries

- Our selector tool helps you to find the most relevant journal

- We provide round the clock customer support

- Convenient online submission

- Thorough peer review

- Inclusion in PubMed and all major indexing services

- Maximum visibility for your research

Submit your manuscript at www.biomedcentral.com/submit

C) Biomed Central 